Published in final edited form as:

Clin Liver Dis. 2016 August ; 20(3): 445-456. doi:10.1016/j.cld.2016.02.004.

\title{
Pathogenesis of Alcoholic Liver Disease
}

\author{
Winston Dunn ${ }^{1}$ and Vijay H. Shah ${ }^{2}$ \\ ${ }^{1}$ Gastroenterology \& Hepatology, The University of Kansas Medical Center, 3901 Rainbow \\ Boulevard, Kansas City, KS USA \\ ${ }^{2}$ Gastroenterology Research Unit, Division of Gastroenterology and Hepatology, Mayo Clinic, \\ Rochester, MN USA
}

\begin{abstract}
Alcoholic Liver Disease includes a broad clinical-histological spectrum from simple steatosis, cirrhosis, acute alcoholic hepatitis with or without cirrhosis to hepatocellular carcinoma as a complication of cirrhosis. The pathogenesis of ALD can be conceptually divided into 1) Ethanol mediated liver injury, 2) Inflammatory Immune response to injury, 3) Intestinal permeability and microbiome changes. Corticosteroids may improve outcomes, but this is controversial and probably only impacts short-term survival. New pathophysiology-based therapies are under study, including IL22, anakinra, and others. These studies provide hope for better future outcomes for this difficult disease.
\end{abstract}

\section{Keywords}

Sterile necrosis; corticosteroids; alcoholic liver disease; alcoholic hepatitis; intestinal permeability; microbiome

\section{Spectrum, Risk Factors, and Comorbidities}

Alcoholic liver disease (ALD) includes a broad spectrum of disorders, such as simple steatosis, cirrhosis, acute alcoholic hepatitis (AH) with or without cirrhosis, and hepatocellular carcinoma (HCC) as a complication of cirrhosis. ALD can also be superimposed on other common liver diseases, including nonalcoholic liver disease (NAFLD) and hepatitis $\mathrm{C}$ virus (HCV) infection, accentuating their prevalence and severity. A large French study looked at 1,604 biopsies on patients admitted for alcoholism or ALD and found normal liver, $14 \%$; steatosis without fibrosis, $29 \%$; some fibrosis \pm steatosis, $20 \%$; steatohepatitis without cirrhosis, $8 \%$; cirrhosis total, $43 \%$; cirrhosis and steatohepatitis, $13 \%{ }^{1}$. An autopsy study of Missouri and Kansas motor vehicle accident victims, who were heavily intoxicated (average blood alcohol concentration, $0.22 \%$ ) at the time of death,

(Corresponding Author) (shah.vijay@mayo.edu). (winstondunnmd@gmail.com).

Publisher's Disclaimer: This is a PDF file of an unedited manuscript that has been accepted for publication. As a service to our customers we are providing this early version of the manuscript. The manuscript will undergo copyediting, typesetting, and review of the resulting proof before it is published in its final citable form. Please note that during the production process errors may be discovered which could affect the content, and all legal disclaimers that apply to the journal pertain.

The authors have nothing to disclose. 
showed that the age-standardized prevalence values were $56 \%$ for hepatic steatosis, $6 \%$ for steatohepatitis, and $18 \%$ for advanced fibrosis ${ }^{2}$.

\section{Risk Factors}

AH usually refers to an acute symptomatic manifestation of histological steatohepatitis. The mouse model by Bin Gao suggested that AH requires " 2 hits", combining chronic heavy alcohol consumption in addition to alcohol binging ${ }^{3}$. In human studies, most subjects reported heavy alcohol use (more than $100 \mathrm{~g} / \mathrm{day}$ ) for two or more decades ${ }^{4}$. The patients are usually 40-60 years of age. Some patients report recent life events as an explanation for the escalated alcohol consumption ${ }^{5}$. Excessive weight is a risk factor for $\mathrm{AH}$, which can results in a combination of both alcoholic and nonalcoholic steatohepatitis ${ }^{1}$. Patients with $\mathrm{HCV}$ are also more likely to develop $\mathrm{AH}$ and severe $\mathrm{AH}^{6}$.

While AH is associated with female sex and an irregular (i.e., binge) pattern of alcohol consumption. Cirrhosis is associated with female sex, heavy alcohol use duration greater than 15 years, and consumption greater than $200 \mathrm{~g}$ /day. PNPLA3 is a novel risk factor for alcoholic cirrhosis. With each additional $\mathrm{G}$ allele, patients are more likely to have alcoholic cirrhosis and to develop alcoholic cirrhosis with a shorter exposure to heavy drinking 7,8 .

In patients with alcoholic cirrhosis, the risk factors for the development of HCC include obesity, diabetes, active alcohol consumption, and viral hepatitis ${ }^{9-11}$. PNPLA3 is also a risk factor for HCC, especially in patients with ALD (and, to a lesser extent, HCV) ${ }^{12}$.

\section{Diagnosis}

Distinguishing an alcoholic basis from a nonalcoholic basis for clinical and histologic steatohepatitis is difficult because of the unreliability of alcohol consumption history. The ALD/NAFLD Index (ANI) uses the mean corpuscular volume (MCV), the aspartate aminotransferase (AST)/alanine aminotransferase (ALT) ratio, the body mass index (BMI), and gender to identify patients with an ALD component ${ }^{13}$. This model has been validated in hospitalized, ambulatory, and pre-transplantation patients, and compares favorably with other traditional and proposed biomarkers. An online calculator is readily available ${ }^{14}$. Acute AH should be distinguished from decompensated alcoholic cirrhosis or acute on chronic liver disease. A breath Biosensor based on trimethylamine and pentane has been developed for the diagnosis of AH. Trimethylamine is generated from the diet and is metabolized in the liver, while pentane is a marker of oxidative stress ${ }^{15}$.

\section{Pathogenesis}

\section{Ethanol-Mediated Liver Injury}

Binge drinking and the NIAAA model-Binge-drinking AH typically occurs in patients with chronic alcohol consumption and a recent history of excessive alcohol use (Figure 1). In a rodent model, ad libitum ethanol delivery through a liquid diet is only sufficient to produce liver pathology limited to steatosis. The mouse model of chronic and binge ethanol feeding (NIAAA model) developed by Gao et al, uses 10 days of ad libitum liquid followed by acute bolus gavage. While either phase alone causes steatosis, combining 
both phases causes steatohepatitis. Therefore, this model may better represent human acute AH ${ }^{16}$. Similarly, effective models have also been developed by the Tsukamoto group, but require invasive intragastric feeding ${ }^{17}$.

Acetaldehyde-In hepatocytes, the primary pathway of ethanol metabolism is through acetaldehyde by alcohol dehydrogenase in the cytosol. Acetaldehyde is metabolized by aldehyde dehydrogenase (ALDH) in the mitochondria. Aldehyde is highly reactive and can form various protein and DNA adducts ${ }^{18}$. A relative deficiency of the ALDH2 isozyme causes the accumulation of acetaldehyde. The incidence of AH is not increased due to aversion to alcohol and a lower prevalence of alcoholism. The minor pathway involves microsomal enzyme oxidation system (MEOS) and generates more reactive oxygen species, resulting in lipid peroxidation, mitochondrial glutathione depletion, and Sadenosylmethionine depletion. The cytochrome CYP2E1 of MEOS is induced in chronic alcoholism, further contributing to oxidative stress and liver injury.

Damage-Associated Molecular Patterns (DAMPs)_Alcohol metabolism leads to oxidative stress and hepatocyte death. Damaged hepatocytes release endogenous DAMPs, which are usually hidden from the extracellular environment. DAMPs activate cellular pattern recognition receptors, which results in sterile inflammation. Features include the production of proinflammatory cytokines, localization of immune cells to the site of injury, and assembly of a cytosolic protein complex machinery termed the "inflammasome", which convey DAMP signals into proinflammatory cytokines (e.g., interleukin [IL]-1).

Metabolism-Steatosis is characterized by the accumulation of triglycerides, phospholipids, and cholesterol esters in hepatocytes. An early study attributed steatosis to the increased ratio of reduced nicotinamide adenine dinucleotide to oxidized nicotinamide adenine dinucleotide, which inhibits $\beta$-oxidation of fatty acids in mitochondria. Recent studies have indicated that alcohol consumption regulates lipid metabolism transcription factors. Alcohol stimulates lipogenesis by the upregulation of sterol regulatory elementbinding transcription factor $1 \mathrm{c}$ (SREBP-1c) ${ }^{19}$. Alcohol can upregulate SREBP-1c directly via its metabolite acetaldehyde, or indirectly via the endoplasmic reticulum stress response, adenosine, endocannabinoids, bacterial translocation, and downstream lipopolysaccharide (LPS) signaling. Alcohol also downregulates the negative regulators of SREBP-1c, including 5 ' adenosine monophosphate-activated protein kinase (AMPK), Sirtuin 1, adiponectin, and signal transducer and activator of transcription 3 (STAT3). Alcohol inhibits fatty acid oxidation by inhibiting the transcriptional activity and DNA-binding ability of peroxisome proliferator-activated receptor alpha (PPAR- $a){ }^{20}$. Alcohol can downregulate PPAR- $\alpha$ directly via its metabolite acetaldehyde, or indirectly via the cytochrome P450 2E1-derived oxidative stress, adenosine, downregulation of adiponectin, and zinc deficiency. Alcohol consumption can also indirectly modify many factors, including hypoxia-inducible factor-1 $(\mathrm{HIF}-1)^{21}, \mathrm{C} 3{ }^{22}, \mathrm{C} 1 \mathrm{qa}, \mathrm{PKC} \varepsilon^{23}$, and inducible nitric oxide synthase ${ }^{24}$, which contributes to the development of steatosis. 


\section{Inflammatory Immune response to Injury}

Innate immune system-Innate immune signaling is involved in the early stage of ALD with simple steatosis even before the onset of inflammation. Endoplasmic reticulum stress activates interferon regulatory factor 3 (IRF3) via the adaptor molecule STRING. IRF3 is phosphorylated (activated) with a single exposure to alcohol, preceding the development of inflammation ${ }^{25}$. Hepatocyte-specific IRF3 is required for the intrinsic (mitochondrial) apoptosis pathway, while Kupffer cell IRF3 deficiency provides only marginal damage from liver damage 26 .

Bacterial overload and increased gut permeability lead to an increased load of bacteriaderived LPS to the portal circulation and liver. LPS interacts with toll-like receptor 4 (TLR4) on Kupffer cells, activating the TIR-domain-containing adapter-inducing interferon- $\beta$ (TRIF)/IRF-3 signaling pathway, leading to the production of proinflammatory cytokines (i.e., tumor necrosis alpha [TNF-a], IL-1, and IL17). Alcohol also activates the complement system $(\mathrm{C} 3, \mathrm{C} 4)$. The interaction between complement and Kupffer cells leads to proinflammatory cytokines (TNF-a) as well as hepatoprotective (IL-6) and cytoprotective (IL-10) cytokines. TNF-a, palmitic acid, the downregulation of proteasome functions, and IL-17 cause hepatocytes (IL-8, CXCL1, and IL-17) and HSCs (IL-8 and CXCL1) to produce chemokines for neutrophil recruitment.

Adaptive immunity—Chronic alcohol leads to lipid peroxidation. Lipid peroxidation products, such as malondialdehyde and 4-hydroxynonenal, can form protein adducts and serve as antigens to activate adaptive immunity. Antibodies against lipid peroxidation adducts and increased numbers of T cells in liver inflammation have been reported ${ }^{20}$.

Immune paralysis-The leading cause of death in AH is overwhelming bacterial infection, leading to multiorgan failure. Paradoxically, immune activity is heightened with inflammation. Programmed cell death protein 1 (PD-1) and T cell immunoglobulin and mucin protein 3 (TIM-3) are inhibitory receptors on T lymphocytes expressed during chronic inflammation and can lead to immune exhaustion. PD-1 and TIM-3 are overexpressed in $\mathrm{AH}$ patients, more so than stable advanced alcoholic cirrhosis patients. The associated dysfunction in neutrophil phagocytosis and oxidative burst in response to Escherichia coli stimulation (in vitro) can be reversed by antibodies against PD-1 and TIM-3 27 .

\section{Intestinal Permeability Changes and Microbiome}

Alcoholic liver disease is associated with bacterial overgrowth and a lower proportion of Bacteroidaceae and probiotic bacteria such as Lactobacillus. Etiologies include small intestinal dysmotility and alterations in bile acid pool ${ }^{28}$. Ethanol disrupts intestinal tight junction integrity ${ }^{39}$. Patients with chronic alcohol abuse with or without ALD have a "leaky gut," as evidenced by a higher level of plasma endotoxin than that in healthy control subjects ${ }^{29}$. In animal models, protection from bacterial overgrowth via genetic mutation ${ }^{30}$ or antibiotics ${ }^{31}$, or the expression of nonfunctional TLR4 (LPS receptor) ${ }^{32}$ can all attenuate alcohol-induced liver injury. 


\section{Zinc}

Zinc deficiency is common in ALD. Animal models have demonstrated that zinc deficiency attenuates alcohol-induced liver injury through various mechanisms, but most importantly, zinc deficiency impairs the intestinal barrier ${ }^{33}$, leading to endotoxin-induced cytokine production. In mouse models, ethanol exposure reduces plasma leptin level ${ }^{34}$, stimulates white adipose tissue lipolysis ${ }^{35,36}$, and inhibits hepatic fatty acid oxidation. Dietary zinc deficiency worsens the alcohol-induced decline of plasma leptin and impairs hepatic fatty acid oxidation ${ }^{37}$. Zinc also has antioxidant properties. Dietary zinc deficiency can also lead to downregulation of antioxidant enzymes, including superoxide dismutase 137 .

\section{Impaired Regeneration}

Usually, liver damage induces mature hepatocytes to proliferate and replace damaged tissue. Given massive or chronic liver damage that is beyond the proliferative capacity of hepatocytes, progenitor cells proliferate to produce a ductular reaction. KRT7 is a marker of ductular reaction in liver disease, and it is typically expressed in liver progenitor cells. Prominin-1 (PROM1) is a marker of progenitor cells from the liver and is considered a marker for hepatic cancer stem cells. Both markers, as measured by real-time PCR in hepatic gene expression analysis, are associated with short-term mortality in AH. In a cohort of 59 patients with biopsy, KRT7, PROM1, MELD score, and ABIC score were independent predictors for 90 -day mortality ${ }^{34}$. In a study that examined explants from AH salvage transplants, a near absence of hepatocyte proliferation was noted ${ }^{25}$. Hepatic progenitor cells were increased, but all expressed laminin (extracellular matrix protein favoring cholangiocyte differentiation) and were therefore inefficient at yielding mature hepatocytes.

\section{Epigenetics and microRNAs (miRNAs)}

miRNAs are short noncoding RNAs that are an average of only 22 nucleotides long. They control the expression of genes involved in cell growth, differentiation, and apoptosis, and are believed to be involved in the pathogenesis of liver disease, particularly cancer. The level of plasma miRNA-155 correlates with liver inflammation induced by alcohol and may be used potentially as a biomarker ${ }^{38}$. Short-term alcohol exposure upregulates miRNA-212 in intestinal epithelial cells, downregulating zonula occludens-1 protein, a protein that regulates intestinal permeability. Alcohol upregulates miRNA-217, which induces lipid synthesis and reduces fatty acid oxidation in the liver via inhibiting the AMPK and sirtuin 1 (SIRT1) pathways ${ }^{39}$. Chronic alcohol also decreases the expression of miRNA 196a and c, which are involved in early regeneration ${ }^{40}$. The expression of liver miRNAs has also been shown to be significantly altered in alcohol-fed mice, but the functions of these miRNAs in the pathogenesis of ALD are not clear.

\section{Protective Mechanisms}

Autophagy-Selective autophagy is a protective mechanism for removing lipid droplets, protein aggregates, and damaged organelles from hepatocytes ${ }^{41}$. In a mouse model, alcohol may activate autophagy in hepatocytes to remove lipid droplets and damaged mitochondria, and therefore, attenuate alcohol-induced steatosis and liver injury. Lipophagy, the autophagy of alcohol-induced lipid droplets, has also been identified ${ }^{42}$. The induction of autophagy 
can potentially be an approach for treating alcohol-induced liver injury ${ }^{43}$. Autophagy in hepatic stellate cells (HSCs), on the other hand, promotes liver fibrosis by using free fatty acids as an energy source ${ }^{44}$

FOXO3-Forkhead Box O3 (FOXO3) plays an important role in the protection against alcohol-induced liver injury through the regulation of autophagy ${ }^{45}$, antioxidants ${ }^{46}$, and proapoptosis-related gene expression. FOXO3 is regulated by post-transcriptional modifications, including phosphorylation by protein kinase B (Akt) and deacetylation by Sirt1. The dephosphorylated and deacetylated forms are retained in the nucleus and can bind to promoters of target genes. Alcohol can potentially affect FOXO3 at multiple levels. Alcohol inhibits Akt phosphorylation and increases the nuclear retention of FOXO3. Alcohol metabolism increases the NADH/NAD ratio, which decreases Sirt1-mediated deacetylation. Resveratrol, a polyphenol antioxidant found in wine, is also a SIRT1 agonist. Combining alcohol and resveratrol (mouse model) can overcome the alcohol-induced Sirt1 inhibition and further increase autophagy and antioxidant-related gene expression.

The FoxO3 mechanism may also explain why patients with HCV do poorly with alcohol abuse. Either alcohol or $\mathrm{HCV}$ alone increases FOXO3 nuclear retention, but the combination has the opposite effect ${ }^{46}$. HCV promotes c-Jun N-terminal kinase (JNK) phosphorylation at Ser574 on FOXO3, which promotes nuclear retention. Alcohol inhibits arginine-methylation of FOXO3, which promotes nuclear export and degradation of the phosphorylated form of $\mathrm{JNK}^{47}$.

\section{HCC Development in ALD}

The general mechanism for the development of HCC includes i) limitation of the regenerative reserve and induction of chromosomal instability by telomeres, ii) cancer induction by the loss of replicative competition due to impaired hepatocyte proliferation, iii) an altered microenvironment promoting tumor cell proliferation, and 4) the loss of cell cycle check point, including resistance to apoptosis and activation of oncogenic pathways. A few of these mechanisms are specific to ALD ${ }^{48}$. Acetaldehyde itself is a carcinogen with mutagenic properties. Alcohol also has an immunosuppressive effect. And, in HCV-infected patients, alcohol can upregulate cancer stem cells ${ }^{49}$.

\section{Current Therapies and Future Approaches}

For more information on specific therapies for AH, please see Paulina K. Phillips and Michael R. Lucey: Acute Alcoholic Hepatitis - Therapy and Srinivasan Dasarathy: Nutrition and Alcoholic Liver Disease: Effects of alcoholism on nutrition, Effects of nutrition on ALD and Nutritional therapies for ALD, in this issue. A few potential therapies that relate to the molecular pathogenesis of ALD will be addressed here.

\section{S-Adenosylmethionine}

Alcohol affects multiple steps in methionine metabolism. S-adenosylmethionine (SAM) is the substrate for the methylation reaction. After donating the methyl group, SAM is converted to S-adenosylhomocysteine (SAH). SAM can be regenerated via a folate- 
dependent or -independent pathway that uses betaine as a substrate. It has been hypothesized that chronic alcohol consumption decreases SAM to the SAH level, leading to mitochondrial damage and endoplasmic reticulum stress. In human studies, the treatment of ALD patients with SAM or placebo has not resulted in any difference in histopathology, AST, ALT, or bilirubin level ${ }^{50-51}$.

\section{Microbiome, LPS, and the Gut Barrier}

Selective intestinal decontamination has been shown to improve liver hemodynamics in decompensated alcoholic cirrhosis ${ }^{52}$. Rifaximin has been shown to improve thrombocytopenia (a marker of portal hypertension) and MELD in alcoholic cirrhosis through the reduction of endotoxemia ${ }^{53}$. Probiotics have been shown to improve neutrophil function, cytokine response, and liver function. These previous studies have conceptually shown the role of microbiome/LPS/gut barrier in ALD, leading to three National Institute of Health (NIH) consortia studies. The first study will focus on the anti-LPS antibody in combination with corticosteroid, the second will study probiotics, and the third will study the efficacy of zinc in restoring the integrity of the gut barrier.

\section{IL-1 Antagonist}

Kupffer cells bear the receptor for DAMPs and LPS, and activate the innate immune system via several cytokines, including TNF-a, IL-6, and IL-1 $\beta$. The IL1 receptor antagonist anakinra will be tested in the NIH consortium ${ }^{54}$.

\section{Inhibition of Apoptosis}

While studies on TNF-a inhibition have failed, the NIH consortium will focus on caspase inhibition in hepatocytes; caspase is a death induction molecule downstream of TNF-a signaling. Caspase inhibition with emricasan may circumvent the immunosuppression and regeneration-blocking effect of TNF-a blockade, and may dampen the innate immune system activation cascade through sterile necrosis and DAMPs release. A phase II clinical trial in nonalcoholic steatohepatitis (NASH) showed that caspase inhibition improved liver enzyme activity 55 .

\section{Farnesoid $X$ receptor (FXR)}

FXR engages in a negative feedback loop for bile acid homeostasis. It negatively regulates lipogenesis through the SREBP1 and liver X receptor (LXR)/SHP axis, and positively regulates fatty acid oxidation through PPARa. This can potentially counteract the steatosis pathophysiology in nonalcoholic steatohepatitis (NASH) and ALD. More importantly, FXR activation may downregulate HSC activation and, therefore, has antifibrotic properties 56 . Currently, FXR agonist studies are still underway for both NASH and alcoholic steatohepatitis.

\section{Interleukin-22}

Interleukin-22 (IL-22) ${ }^{57}$ is produced by Th17 cells and natural killer cells. The biological effect is mediated mainly via activation of the STAT3 signaling pathway through the binding of IL-22R1 and IL-10R2. In a murine model of chronic-binge alcohol feeding, treatment 
with IL-22 recombinant protein activates hepatic STAT3 and ameliorates alcoholic fatty liver, liver injury, and hepatic oxidative stress. In patients with AH, expression of IL-22R1 is upregulated, while IL-22 is undetectable, suggesting that IL-22 treatment could be a potential therapeutic option.

\section{Osteopontin}

Osteopontin is a chemoattractant for polymorphonuclear leukocytes (PMN) and promotes fibrosis in NASH. The role of osteopontin has been controversial in ALD depending on the experimental model. Lazaro et al used the NIAAA model of acute AH superimposed on a background of severe steatosis, and demonstrated that osteopontin deficiency does not prevent, but rather promotes $\mathrm{AH}^{58}$, suggesting a potential therapeutic target.

\section{Cannabinoid}

Cannabinoid receptor type 1 (CB1) is a promoter of fibrosis, while Cannabinoid receptor type 2 (CB2) is an inhibitor ${ }^{59}$. In response to alcohol feeding, steatosis and fibrogenesis are increased in CB2 receptor-deficient mice and decreased in CB1 receptor knockouts ${ }^{60}$. Rimonabant is a CB1 antagonist used in Europe for the treatment of obesity and metabolic syndrome, before it was withdrawn from the market for its adverse effect of depression. Non-psychoactive cannabinoids are currently being developed ${ }^{61}$.

\section{REFERENCES}

1. Naveau S, Giraud V, Borotto E, Aubert A, Capron F, Chaput JC. Excess weight risk factor for alcoholic liver disease. Hepatology. Jan; 1997 25(1):108-111. [PubMed: 8985274]

2. Dunn W, Zeng Z, O'Neil M, et al. The interaction of rs738409, obesity, and alcohol: a populationbased autopsy study. The American journal of gastroenterology. Nov; 2012 107(11):1668-1674. [PubMed: 23032985]

3. Deng QG, She H, Cheng JH, et al. Steatohepatitis induced by intragastric overfeeding in mice. Hepatology. Oct; 2005 42(4):905-914. [PubMed: 16175602]

4. Cohen SM, Ahn J. Review article: the diagnosis and management of alcoholic hepatitis. Alimentary pharmacology \& therapeutics. Jul; 2009 30(1):3-13. [PubMed: 19416132]

5. Choi G, Runyon BA. Alcoholic hepatitis: a clinician's guide. Clinics in liver disease. May; 2012 16(2):371-385. [PubMed: 22541704]

6. Punzalan CS, Bukong TN, Szabo G. Alcoholic hepatitis and HCV interactions in the modulation of liver disease. Journal of viral hepatitis. Mar.2015 :6. [PubMed: 25560839]

7. Tian C, Stokowski RP, Kershenobich D, Ballinger DG, Hinds DA. Variant in PNPLA3 is associated with alcoholic liver disease. Nature genetics. Jan; 2010 42(1):21-23. [PubMed: 19946271]

8. Burza MA, Molinaro A, Attilia ML, et al. PNPLA3 I148M (rs738409) genetic variant and age at onset of at-risk alcohol consumption are independent risk factors for alcoholic cirrhosis. Liver international : official journal of the International Association for the Study of the Liver. Apr; 2014 34(4):514-520. [PubMed: 24102786]

9. Saunders D, Seidel D, Allison M, Lyratzopoulos G. Systematic review: the association between obesity and hepatocellular carcinoma - epidemiological evidence. Alimentary pharmacology \& therapeutics. May; 2010 31(10):1051-1063. [PubMed: 20175765]

10. Loomba R, Yang HI, Su J, et al. Synergism between obesity and alcohol in increasing the risk of hepatocellular carcinoma: a prospective cohort study. American journal of epidemiology. Feb 15; 2013 177(4):333-342. [PubMed: 23355498]

11. Donato F, Tagger A, Gelatti U, et al. Alcohol and hepatocellular carcinoma: the effect of lifetime intake and hepatitis virus infections in men and women. American journal of epidemiology. Feb 15; 2002 155(4):323-331. [PubMed: 11836196] 
12. Trepo E, Nahon P, Bontempi G, et al. Association between the PNPLA3 (rs738409 C>G) variant and hepatocellular carcinoma: Evidence from a meta-analysis of individual participant data. Hepatology. Jun; 2014 59(6):2170-2177. [PubMed: 24114809]

13. Dunn W, Angulo P, Sanderson S, et al. Utility of a new model to diagnose an alcohol basis for steatohepatitis. Gastroenterology. Oct; 2006 131(4):1057-1063. [PubMed: 17030176]

14. http://www.mayoclinic.org/medical-professionals/model-end-stage-liver-disease/alcoholic-liverdisease-nonalcoholic-fatty-liver-disease-index. http://www.mayoclinic.org/medical-professionals/ model-end-stage-liver-disease/alcoholic-liver-disease-nonalcoholic-fatty-liver-disease-index

15. Hanouneh IA, Zein NN, Cikach F, et al. The breathprints in patients with liver disease identify novel breath biomarkers in alcoholic hepatitis. Clinical gastroenterology and hepatology : the official clinical practice journal of the American Gastroenterological Association. Mar; 2014 12(3):516-523. [PubMed: 24036050]

16. Arteel GE. Build a better mouse model, and the world will beat a path to your door. Hepatology. Nov; 2013 58(5):1526-1528. [PubMed: 23813457]

17. Tsukamoto H, French SW, Reidelberger RD, Largman C. Cyclical pattern of blood alcohol levels during continuous intragastric ethanol infusion in rats. Alcoholism, clinical and experimental research. Jan-Feb;1985 9(1):31-37.

18. Setshedi M, Wands JR, Monte SM. Acetaldehyde adducts in alcoholic liver disease. Oxidative medicine and cellular longevity. May-Jun;2010 3(3):178-185. [PubMed: 20716942]

19. Ji C, Chan C, Kaplowitz N. Predominant role of sterol response element binding proteins (SREBP) lipogenic pathways in hepatic steatosis in the murine intragastric ethanol feeding model. Journal of hepatology. Nov; 2006 45(5):717-724. [PubMed: 16879892]

20. Li HH, Tyburski JB, Wang YW, et al. Modulation of fatty acid and bile acid metabolism by peroxisome proliferator-activated receptor alpha protects against alcoholic liver disease. Alcoholism, clinical and experimental research. Jun; 2014 38(6):1520-1531.

21. Nath B, Levin I, Csak T, et al. Hepatocyte-specific hypoxia-inducible factor-1alpha is a determinant of lipid accumulation and liver injury in alcohol-induced steatosis in mice. Hepatology. May; 2011 53(5):1526-1537. [PubMed: 21520168]

22. Pritchard MT, McMullen MR, Stavitsky AB, et al. Differential contributions of C3, C5, and decayaccelerating factor to ethanol-induced fatty liver in mice. Gastroenterology. Mar; 2007 132(3): 1117-1126. [PubMed: 17383432]

23. Kaiser JP, Beier JI, Zhang J, et al. PKCepsilon plays a causal role in acute ethanol-induced steatosis. Archives of biochemistry and biophysics. Feb; 2009 482(1-2):104-111. [PubMed: 19022218]

24. McKim SE, Gabele E, Isayama F, et al. Inducible nitric oxide synthase is required in alcoholinduced liver injury: studies with knockout mice. Gastroenterology. Dec; 2003 125(6):1834-1844. [PubMed: 14724835]

25. Petrasek J, Iracheta-Vellve A, Csak T, et al. STING-IRF3 pathway links endoplasmic reticulum stress with hepatocyte apoptosis in early alcoholic liver disease. Proceedings of the National Academy of Sciences of the United States of America. Oct 8; 2013 110(41):16544-16549. [PubMed: 24052526]

26. Petrasek J, Dolganiuc A, Csak T, et al. Interferon regulatory factor 3 and type I interferons are protective in alcoholic liver injury in mice by way of crosstalk of parenchymal and myeloid cells. Hepatology. Feb; 2011 53(2):649-660. [PubMed: 21274885]

27. Markwick LJ, Riva A, Ryan JM, et al. Blockade of PD1 and TIM3 restores innate and adaptive immunity in patients with acute alcoholic hepatitis. Gastroenterology. Mar; 2015 148(3):590-602. e510. [PubMed: 25479137]

28. Kakiyama G, Pandak WM, Gillevet PM, et al. Modulation of the fecal bile acid profile by gut microbiota in cirrhosis. Journal of hepatology. May; 2013 58(5):949-955. [PubMed: 23333527]

29. Elamin EE, Masclee AA, Dekker J, Jonkers DM. Ethanol metabolism and its effects on the intestinal epithelial barrier. Nutrition reviews. Jul; 2013 71(7):483-499. [PubMed: 23815146]

30. Parlesak A, Schafer C, Schutz T, Bode JC, Bode C. Increased intestinal permeability to macromolecules and endotoxemia in patients with chronic alcohol abuse in different stages of 
alcohol-induced liver disease. Journal of hepatology. May; 2000 32(5):742-747. [PubMed: 10845660]

31. Adachi Y, Moore LE, Bradford BU, Gao W, Thurman RG. Antibiotics prevent liver injury in rats following long-term exposure to ethanol. Gastroenterology. Jan; 1995 108(1):218-224. [PubMed: 7806045]

32. Uesugi T, Froh M, Arteel GE, Bradford BU, Thurman RG. Toll-like receptor 4 is involved in the mechanism of early alcohol-induced liver injury in mice. Hepatology. Jul; 2001 34(1):101-108. [PubMed: 11431739]

33. Zhong W, McClain CJ, Cave M, Kang YJ, Zhou Z. The role of zinc deficiency in alcohol-induced intestinal barrier dysfunction. American journal of physiology. Gastrointestinal and liver physiology. May; 2010 298(5):G625-633. [PubMed: 20167873]

34. Tan X, Sun X, Li Q, et al. Leptin deficiency contributes to the pathogenesis of alcoholic fatty liver disease in mice. The American journal of pathology. Oct; 2012 181(4):1279-1286. [PubMed: 22841822]

35. Zhong W, Zhao Y, Tang Y, et al. Chronic alcohol exposure stimulates adipose tissue lipolysis in mice: role of reverse triglyceride transport in the pathogenesis of alcoholic steatosis. The American journal of pathology. Mar; 2012 180(3):998-1007. [PubMed: 22234172]

36. Wei X, Shi X, Zhong W, et al. Chronic alcohol exposure disturbs lipid homeostasis at the adipose tissue-liver axis in mice: analysis of triacylglycerols using high-resolution mass spectrometry in combination with in vivo metabolite deuterium labeling. PloS one. Aug.2013 (2):e55382. [PubMed: 23405143]

37. Zhong W, Zhao Y, Sun X, Song Z, McClain CJ, Zhou Z. Dietary zinc deficiency exaggerates ethanol-induced liver injury in mice: involvement of intrahepatic and extrahepatic factors. PloS one. 2013; 8(10):e76522. [PubMed: 24155903]

38. Bala S, Petrasek J, Mundkur S, et al. Circulating microRNAs in exosomes indicate hepatocyte injury and inflammation in alcoholic, drug-induced, and inflammatory liver diseases. Hepatology. Nov; 2012 56(5):1946-1957. [PubMed: 22684891]

39. Yin H, Hu M, Zhang R, Shen Z, Flatow L, You M. MicroRNA-217 promotes ethanol-induced fat accumulation in hepatocytes by down-regulating SIRT1. The Journal of biological chemistry. Mar 23; 2012 287(13):9817-9826. [PubMed: 22308024]

40. Dippold RP, Vadigepalli R, Gonye GE, Patra B, Hoek JB. Chronic ethanol feeding alters miRNA expression dynamics during liver regeneration. Alcoholism, clinical and experimental research. Jan; 2013 37(Suppl 1):E59-69.

41. Czaja MJ, Ding WX, Donohue TM Jr. et al. Functions of autophagy in normal and diseased liver. Autophagy. Aug; 2013 9(8):1131-1158. [PubMed: 23774882]

42. Ronis MJ, Mercer KE, Gannon B, et al. Increased 4-hydroxynonenal protein adducts in male GSTA4-4/PPAR-alpha double knockout mice enhance injury during early stages of alcoholic liver disease. American journal of physiology. Gastrointestinal and liver physiology. Mar 1; 2015 308(5):G403-415. [PubMed: 25501545]

43. Ding WX. Induction of autophagy, a promising approach for treating liver injury. Hepatology. Jan; 2014 59(1):340-343. [PubMed: 23775596]

44. Hernandez-Gea V, Ghiassi-Nejad Z, Rozenfeld R, et al. Autophagy releases lipid that promotes fibrogenesis by activated hepatic stellate cells in mice and in human tissues. Gastroenterology. Apr; 2012 142(4):938-946. [PubMed: 22240484]

45. Ni HM, Du K, You M, Ding WX. Critical role of FoxO3a in alcohol-induced autophagy and hepatotoxicity. The American journal of pathology. Dec; 2013 183(6):1815-1825. [PubMed: 24095927]

46. Tumurbaatar B, Tikhanovich I, Li Z, et al. Hepatitis C and alcohol exacerbate liver injury by suppression of FOXO3. The American journal of pathology. Dec; 2013 183(6):1803-1814. [PubMed: 24225087]

47. Tikhanovich I, Kuravi S, Campbell RV, et al. Regulation of FOXO3 by phosphorylation and methylation in hepatitis C virus infection and alcohol exposure. Hepatology. Jan; 2014 59(1):5870. [PubMed: 23857333] 
48. McKillop IH, Schrum LW. Role of alcohol in liver carcinogenesis. Seminars in liver disease. May; 2009 29(2):222-232. [PubMed: 19387921]

49. Machida K, Tsukamoto H, Mkrtchyan H, et al. Toll-like receptor 4 mediates synergism between alcohol and HCV in hepatic oncogenesis involving stem cell marker Nanog. Proceedings of the National Academy of Sciences of the United States of America. Feb 3; 2009 106(5):1548-1553. [PubMed: 19171902]

50. Le MD, Enbom E, Traum PK, Medici V, Halsted CH, French SW. Alcoholic liver disease patients treated with S-adenosyl-L-methionine: an in-depth look at liver morphologic data comparing pre and post treatment liver biopsies. Experimental and molecular pathology. Oct; 2013 95(2):187191. [PubMed: 23886644]

51. Medici V, Virata MC, Peerson JM, et al. S-adenosyl-L-methionine treatment for alcoholic liver disease: a double-blinded, randomized, placebo-controlled trial. Alcoholism, clinical and experimental research. Nov; 2011 35(11):1960-1965.

52. Seo YS, Shah VH. The role of gut-liver axis in the pathogenesis of liver cirrhosis and portal hypertension. Clin Mol Hepatol. Dec; 2012 18(4):337-346. [PubMed: 23323248]

53. Kalambokis GN, Mouzaki A, Rodi M, Tsianos EV. Rifaximin improves thrombocytopenia in patients with alcoholic cirrhosis in association with reduction of endotoxaemia. Liver international : official journal of the International Association for the Study of the Liver. Mar; 2012 32(3):467-475. [PubMed: 22098272]

54. Petrasek J, Bala S, Csak T, et al. IL-1 receptor antagonist ameliorates inflammasome-dependent alcoholic steatohepatitis in mice. J Clin Invest. Oct; 2012 122(10):3476-3489. [PubMed: 22945633]

55. Ratziu V, Sheikh MY, Sanyal AJ, et al. A phase 2, randomized, double-blind, placebo-controlled study of GS-9450 in subjects with nonalcoholic steatohepatitis. Hepatology. Feb; 2012 55(2):419428. [PubMed: 22006541]

56. Verbeke L, Farre R, Trebicka J, et al. Obeticholic acid, a farnesoid X receptor agonist, improves portal hypertension by two distinct pathways in cirrhotic rats. Hepatology. Jun; 2014 59(6):22862298. [PubMed: 24259407]

57. Ki SH, Park O, Zheng M, et al. Interleukin-22 treatment ameliorates alcoholic liver injury in a murine model of chronic-binge ethanol feeding: role of signal transducer and activator of transcription 3. Hepatology. Oct; 2010 52(4):1291-1300. [PubMed: 20842630]

58. Lazaro R, Wu R, Lee S, et al. Osteopontin deficiency does not prevent but promotes alcoholic neutrophilic hepatitis in mice. Hepatology. Jan; 2015 61(1):129-140. [PubMed: 25132354]

59. Basu PP, Aloysius MM, Shah NJ, Brown RS Jr. Review article: the endocannabinoid system in liver disease, a potential therapeutic target. Alimentary pharmacology \& therapeutics. Apr; 2014 39(8):790-801. [PubMed: 24612021]

60. Trebicka J, Racz I, Siegmund SV, et al. Role of cannabinoid receptors in alcoholic hepatic injury: steatosis and fibrogenesis are increased in $\mathrm{CB} 2$ receptor-deficient mice and decreased in $\mathrm{CB} 1$ receptor knockouts. Liver international : official journal of the International Association for the Study of the Liver. Jul; 2011 31(6):860-870. [PubMed: 21645218]

61. Silvestri C, Paris D, Martella A, et al. Two non-psychoactive cannabinoids reduce intracellular lipid levels and inhibit hepatosteatosis. Journal of hepatology. Jun; 2015 62(6):1382-1390. [PubMed: 25595882] 


\section{Key Points}

1. Alcoholic liver disease, especially alcoholic hepatitis, continues to be a major cause of liver-related morbidity and mortality.

2. Alcohol and its metabolic byproducts, including acetaldehyde, contribute to liver injury.

3. Liver injury leads to inflammation in alcoholic hepatitis through a classical "sterile necrosis" response.

4. Enhanced gut permeability and changes in microbiome also contribute to alcoholic liver disease.

5. New pathophysiology-based therapies are undergoing evaluation in patients. 


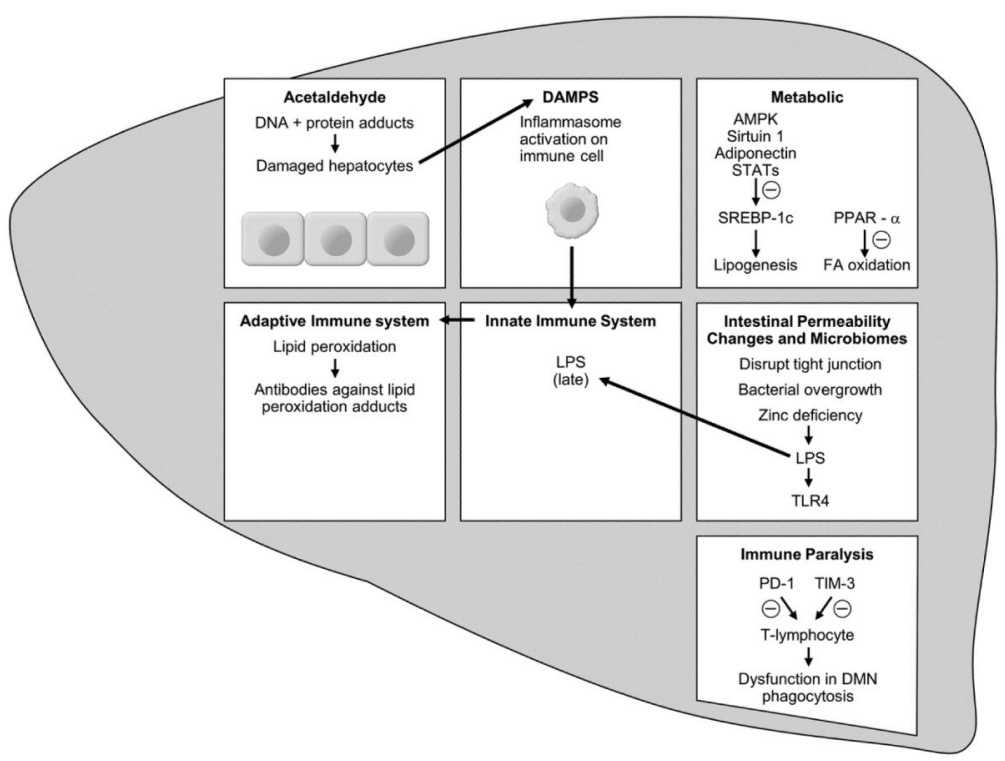

Figure 1.

Mechanisms of alcoholic liver disease. Alcohol contributes to liver injury through a multitude of ways as depicted. Alcohol is metabolized to acetaldehyde; both alcohol and acetaldehyde have toxic effects on hepatocytes. Damaged hepatocytes in turn release DAMPs that recruit innate and adaptive immune cells that perpetuate further liver injury. Earlier alcohol lesion of steatosis is mediated by effects of alcohol or lipogenesis and fatty acid oxidation. Alcohol also has direct effects on intestinal microbiome and gut permeability that allows bacterial products to reach liver, and further stimulate immune response and liver injury. Finally, despite immune stimulation, the immune response is ineffective in combating infection, termed immune paralysis. 